\title{
Influence of morphological texture on stresses analysis by X-ray and neutron diffraction: accounting for extreme morphologies
}

\author{
Viwanou Hounkpati ${ }^{1, a}$, Sylvain Fréour ${ }^{1, b}$, David Gloaguen ${ }^{1, c}$ \\ and Vincent Legrand ${ }^{1, d}$
${ }^{1}$ Université de Nantes, Centrale Nantes, Institut de Recherche en Génie Civil et Mécanique (UMR CNRS 6183), Equipe Etat Mécanique et Microstructure, 58 rue Michel Ange, BP 420, 44606, Saint-Nazaire cedex, France \\ aviwanou.hounkpati@univ-nantes.fr, bsylvain.freour@univ-nantes.fr, \\ cdavid.gloaguen@univ-nantes.fr, dvincent.legrand@univ-nantes.fr
}

\begin{abstract}
Keywords: Self-consistent model, residual stress, textures, morphologies, X-ray diffraction, neutron diffraction.

Abstract. This work investigates, by diffraction methods, the morphological texture influence on the residual stress analysis in polycrystals having cubic or hexagonal symmetries. Different extreme crystallite morphologies (sphere, disc and fiber, with their principal axes aligned along common directions) were considered in the present study. In a second part, crystallographic textures were accounted for, also, enabling to reflect the combined effect of the simultaneous occurrence of morphological and crystallographic textures. A stronger influence of morphological texture than that of the crystallographic texture in terms of stresses was observed. The main purpose of this work is to make the best choice of lattice planes (hkl) used for residual stress analysis, in elasticity, depending on the morphological (and crystallographic) texture of the polycristal.
\end{abstract}

\section{Introduction}

Scale transition models proposed by Voigt, Reuss, Neerfeld-Hill or Eshelby-Kröner are traditionally used to describe the distribution of stresses and strains over the differently oriented grains of a mechanically stressed polycrystals [1]. With these models, polycrystalline materials are usually represented by an isotropic morphologic microstructure (i.e. equiaxed or spherical grains are considered). Thus, in the absence of crystallographic texture, polycrystals are macroscopically elastically isotropic. This is not generally the case, even in the absence of crystallographic texture. There are many crystalline materials with very marked morphological microstructures: the case for example of thin solid films with columnar grains has been extensively described in the literature [2].

It appears obvious that polycrystals with a morphological texture have anisotropic macroscopic properties even in the absence of crystallographic texture. The classical Eshelby-Kröner selfconsistent model enables to take into account non-spherical grains. Owing to this model, an ideal morphological texture can be taken into account with (identical) ellipsoidal inclusions whose principal axes $\left(a_{1}, a_{2}, a_{3}\right)$ are aligned preferentially along certain directions in the specimen. It is precisely this preferential alignment of non-spherical grains which leads to an anisotropic macroscopic behavior. Following this line of reasoning, we will consider, in the present work, polycrystals consisting of fiber or disc-shaped grains aligned preferentially along certain directions in the specimen (extreme morphological textures). The main purpose is to show the possible influence of the extremely anisotropic behavior induced at the scale of the diffracting volume by grain-shape in the context of stress analysis by diffraction methods (X-ray or neutron diffraction); we will be interested in the $\varepsilon_{\varphi \psi}$-vs.- $\sin ^{2} \psi$ diagrams and stress states which are traditionally deducted from them. 


\section{Eshelby-Kröner self-consistent modeling}

Accounting for an extreme morphological texture. Eshelby-Kröner self-consistent model is a very relevant model for describing the elastic behavior of crystalline aggregates because it estimates accurately the interactions and effects of intergranular heterogeneities. Apart from the materials consisting of equiaxed grains, a grain-shape texture can also be incorporated in this approach by considering ellipsoidal inclusions, provided that they have the same geometrical orientation in the specimen frame of reference. In this case, the local strain (at the grain scale) $\varepsilon^{\mathrm{II}}$ can be obtained classically through the strain localization A tensor:

$$
\varepsilon^{\mathrm{II}}(\Omega)=[\mathrm{I}+\mathrm{E}:(\mathrm{c}(\Omega)-\mathrm{C})]^{-1}: \varepsilon^{\mathrm{I}}=\mathrm{A}(\Omega): \varepsilon^{\mathrm{I}}
$$

where $\mathrm{c}$ and $\mathrm{C}$ are respectively the mesoscopic and the macroscopic stiffness tensors; I represents the fourth order identity tensor. $\varepsilon^{\mathrm{I}}$ is the average macroscopic strain experienced by the polycrystal. $\mathrm{A}: \mathrm{B}$ denotes the double scalar product $\mathrm{A}_{\mathrm{ijk} l} \mathrm{~B}_{\mathrm{klmn}}$ using the Einstein summation convention. We describe the orientations of a crystallite within a polycrystalline sample by specification of the rotations $\Omega\left(\varphi_{1}, \phi, \varphi_{2}\right)$ which relate the sample to the crystal referential system. $\varphi_{1}, \phi, \varphi_{2}$ are the three Eulerian angles [3]. E is the so-called Morris tensor, which expresses the interaction between an inclusion (grain) with a given morphology and the Homogeneous Equivalent Medium. The Morris tensor E can be calculated for the case of an ellipsoidal inclusion (grain) shape as follows [3]:

$$
\begin{aligned}
& \mathrm{E}_{\mathrm{ijkl}}=\frac{1}{4 \pi} \int_{0}^{\pi} \sin \theta \mathrm{d} \theta \int_{0}^{2 \pi} \gamma_{\mathrm{ikjl}} \mathrm{d} \phi=\mathrm{S}_{\mathrm{ijkl}}^{\mathrm{Esh}} \mathrm{C}_{\mathrm{ijkl}}^{-1} \\
& \text { with } \gamma_{\mathrm{ikjl}}=\mathrm{K}_{\mathrm{ik}}^{-1}(\xi) \xi_{\mathrm{j}} \xi_{1}, \mathrm{~K}_{\mathrm{jp}}(\xi)=\mathrm{C}_{\mathrm{ijpl}} \xi_{\mathrm{i}} \xi_{1}, \xi_{1}=\frac{\sin \theta \cos \phi}{\mathrm{a}_{1}}, \xi_{2}=\frac{\sin \theta \sin \phi}{\mathrm{a}_{2}} \text { and } \xi_{3}=\frac{\cos \theta}{\mathrm{a}_{3}}
\end{aligned}
$$

where $S^{\text {Esh }}$ is the Eshelby tensor; the $a_{i}$ are the lengths of the principal axes of the ellipsoid, used to describe grains shape.

Application to stress analysis by diffraction. Diffraction methods like the well-known " $\sin ^{2} \psi$ method" [1] for determining residual or internal stresses in polycrystalline materials are based on the measurement of lattice spacings of the (hkl) planes in the crystallites contained in the diffracting volume. The diffraction geometry is shown in Fig. 1. The direction of the diffraction vector $\mathrm{n}(\varphi, \psi)=(\sin \varphi \cos \psi, \sin \varphi \sin \psi, \cos \psi)$, is usually identified by the $\varphi$ and $\psi$ angles, where $\psi$ is the declination angle and $\varphi$ denotes the rotation of the specimen around the specimen surface normal. $\mathrm{L}_{1}^{\prime}$ is the stress measurement direction. $\left(\mathrm{S}_{1}, \mathrm{~S}_{2}, \mathrm{~S}_{3}\right)$ and $\left(\mathrm{L}_{1}, \mathrm{~L}_{2}, \mathrm{~L}_{3}\right)$ are respectively the sample and the laboratory frame of reference.

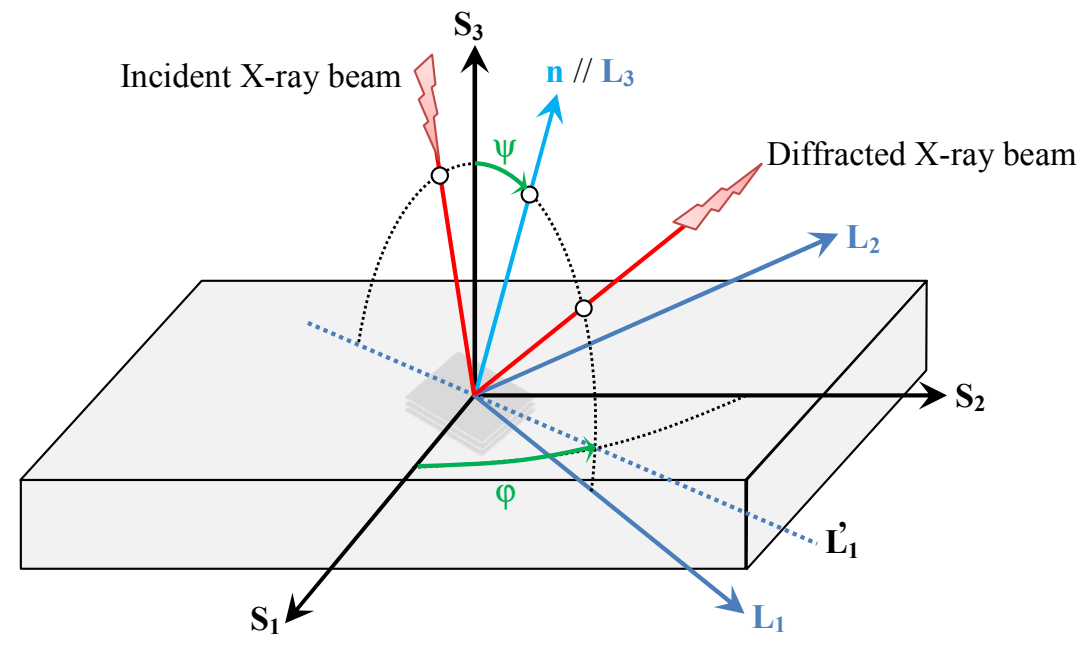

Figure 1 : The diffraction geometry 
The lattice strain $\varepsilon_{\varphi \psi}$ measured by diffraction can be obtained as the average of the second order lattice strains over the diffracting grains for the considered (hkl) plane along the direction of the diffraction vector $n$. Using Eshelby-Kröner formalism (Eq. 1), $\varepsilon_{\varphi \psi}$ is given by the relation

$$
\varepsilon_{\varphi \psi}=\left\langle\varepsilon^{\mathrm{II}}(\varphi, \psi, \mathrm{hkl})\right\rangle_{\mathrm{V}_{\mathrm{d}}}=\mathrm{n}(\varphi, \psi) \cdot\left[[\mathrm{I}+\mathrm{E}:(\mathrm{c}(\Omega)-\mathrm{C})]^{-1}\right\rangle_{\mathrm{V}_{\mathrm{d}}}: \varepsilon^{\mathrm{I}} \cdot{ }^{\mathrm{t}} \mathrm{n}(\varphi, \psi)
$$

where ${ }^{t} \mathrm{n}$ is the transpose of $\mathrm{n}$ and \langle\rangle$_{\mathrm{V}_{\mathrm{d}}}$ stands for the average over the diffracting volume $\mathrm{V}_{\mathrm{d}}$.

In the general case of a textured material (crystallographically or morphologically), the dependence of the measured lattice strains on the macroscopic stresses $\sigma^{\mathrm{I}}$ over diffracting grains is described by the main relationship for residual stress determination, using the X-ray stress factors $F_{\mathrm{ij}}[1]$ :

$$
\varepsilon_{\varphi \psi}=\mathrm{F}_{\mathrm{ij}}(\varphi, \psi, \mathrm{hkl}) \sigma_{\mathrm{ij}}^{\mathrm{I}}
$$

For non-textured materials, the stress factors stand for a combination of the traditional X-ray Elasticity Constants (XECs), $1 / 2 \mathrm{~S}_{2}(\mathrm{hkl})$ and $\mathrm{S}_{1}(\mathrm{hkl})$ :

$$
\mathrm{F}_{\mathrm{ij}}(\varphi, \psi, \mathrm{hkl})=\frac{1}{2} \mathrm{~S}_{2}(\mathrm{hkl}) \mathrm{n}_{\mathrm{i}}(\varphi, \psi) \mathrm{n}_{\mathrm{j}}(\varphi, \psi)+\mathrm{S}_{1}(\mathrm{hkl}) \delta_{\mathrm{ij}}
$$

where $\delta_{\mathrm{ij}}$ is the Kronecker symbol. Using the previous relation, Eq. 5 becomes, for a non-textured, macroscopically isotropic polycrystalline material, by considering the simplifying case corresponding to a tensile test along the $S_{1}$ axis $\left(\varphi=0^{\circ}\right)$ :

$$
\varepsilon_{\varphi \psi}=\mathrm{F}_{11}\left(0^{\circ}, \psi, h \mathrm{kl}\right) \sigma_{11}^{\mathrm{I}}=\frac{1}{2} \mathrm{~S}_{2}(\mathrm{hkl}) \cdot \sigma_{11}^{\mathrm{I}} \sin ^{2} \psi+\mathrm{S}_{1}(\mathrm{hkl}) \cdot \sigma_{11}^{\mathrm{I}}
$$

$\varepsilon_{\varphi \psi}$ plotted versus $\sin ^{2} \psi$ for macroscopically isotropic materials is therefore a straight line. Its slope is proportional to $\sigma_{11}^{\mathrm{I}}$.

Because the stress factors are much more difficult to determine than the XECs, experimentally as by modelling, XECs are often used instead of the stress factors $F_{i j}$ [4]. Thus, significant deviations between the real stress state of the material and the one determined experimentally by diffraction methods may occur. Because of the dependence between the stress factors and the lattice planes, these deviations will vary according to the (hkl) lattice plane analysed. The main interest of the present contribution is to quantify these deviations for a wide range of (hkl) lattice planes in order to propose a better choice of the (hkl) lattice planes according to the morphological texture of the material.

\section{Influence of morphological texture on stress analysis in diffraction}

Using Eq. 4, we simulated uniaxial tensile tests and plotted the evolution $\varepsilon_{\varphi \psi}$-vs.- $\sin ^{2} \psi$ diagrams for different polycrystals with cubic or hexagonal symmetry (aluminum, beryllium, copper, zirconium, titanium, cadmium, alpha-iron and gamma-iron), experiencing a macroscopic stress $\sigma_{11}^{\mathrm{I}}=100 \mathrm{MPa}$. An isotropic crystallographic texture has been used by considering 10000 grains with random crystallographic orientations, to highlight the influence of the morphological texture only. We distinguished two cases for the purpose of simulating $\varepsilon_{\varphi \psi}$-vs.- $\sin ^{2} \psi$ curves: X-ray and neutron diffraction. The X-ray diffraction is simulated with 21 tilt angles $\psi$ ranging from $-60^{\circ}$ to $60^{\circ}$. The neutron diffraction is simulated with 31 tilt angles $\psi$ varying between -90 and $90^{\circ}$. These are typical values used, respectively, in X-ray and neutron diffraction according to reference [1]. The morphology of the crystallites has been described by a shape parameter denoted $\eta$, which is defined as the ratio of the principal axis of the ellipsoid $\left(a_{3}\right)$ and the secondary axes $\left(a_{1}\right.$ or $\left.a_{2}\right)$ of the ellipsoid $\left(\eta=a_{3} / a_{1}=a_{3} / a_{2}\right)$ (see Fig. 2 for the definition of fiber and disc geometries). Shape parameters used to describe the extreme morphological textures investigated in this work are 1, 100 and 0.01 for spherical grains, fiber- and disc- shaped inclusions, respectively. To account for the 
influence of the morphological orientation of the grains, an uniaxial tensile test along $\mathrm{S}_{1}$ axis (loading direction) was simulated by varying the morphological orientation of the grains in the sample (see Fig. 2). For each considered morphology, three different simulations are then performed:

- first, when grain principal axes $\left(a_{3}\right)$ are aligned preferentially along the loading direction $\left(a_{3} / / S_{1}\right)$, - secondly, when the $\left(\mathrm{a}_{1}\right)$ axes of the grains are aligned preferentially along the loading direction and $\left(a_{2}\right)$ perpendicular to the surface of the specimen $\left(a_{1} / / S_{1}, a_{2} \perp\right.$ surface),

- and finally, when the $\left(a_{1}\right)$ axes of the grains are aligned preferentially along the loading direction and $\left(a_{3}\right)$ perpendicular to the surface of the specimen $\left(a_{1} / / S_{1}, a_{3} \perp\right.$ surface); this latter corresponds to the case of thin films with columnar grains, when a fiber texture is considered.
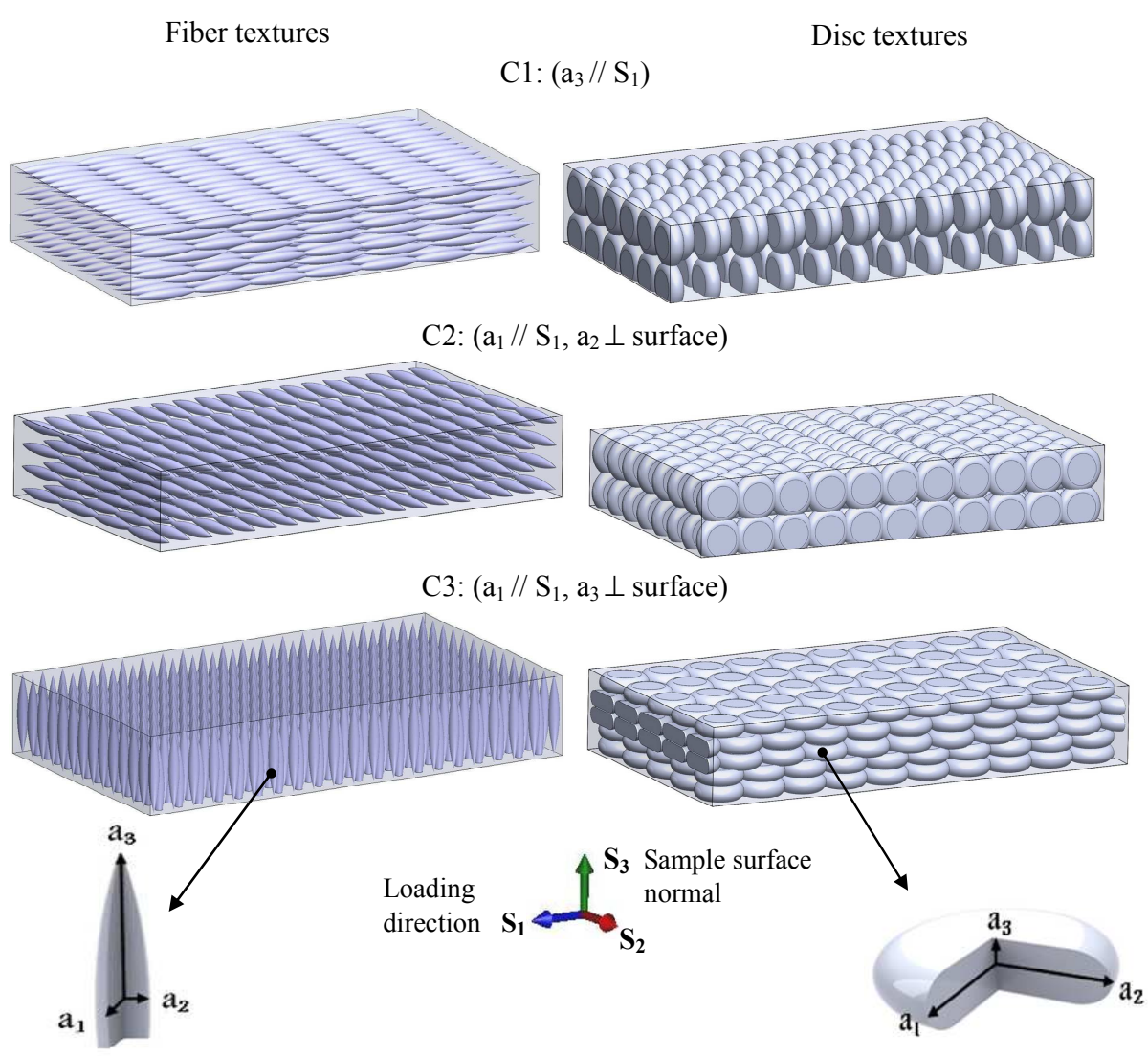

Figure 2 : Different morphological orientations of the grains in the samples.

In the remainder of the paper and for more clarity, the above cases have been denoted $\mathrm{C} 1, \mathrm{C} 2$ and $\mathrm{C} 3$, respectively. Texture effects on stress analysis by diffraction are very low when the single crystal of the material is almost elastically isotropic. For this reason, only the results obtained for the zinc $(\mathrm{Zn})$ and the gamma-iron $(\boldsymbol{\gamma}-\mathrm{Fe})$ were presented. These materials exhibit highly anisotropic elastic properties at mesoscopic level. Several lattice-planes commonly used to perform lattice strains measurements in diffraction [1,5] have been investigated: (200), (220), (222), (311), (420), (331), (511) and (844) for the gamma-iron and (10.0), (00.4), (11.0), (20.1), (20.3), (11.4), (21.3), (30.2) and (10.4) for the zinc. The elastic strains $\varepsilon_{\varphi \psi}$ have been plotted as a function of $\sin ^{2} \psi$ in Fig. 3 for (200) plane of the gamma-iron and (00.4) plane of the zinc, by taking into account the different morphological orientations of the grains defined in Fig. 2. We note that the anisotropy introduced by the morphological texture has greatly changed the $\varepsilon_{\varphi \psi}$-vs.- $\sin ^{2} \psi$ distributions. Actually more or less pronounced oscillations can be observed, depending on the lattice plane analysed. For a given extreme grain morphology (i.e. for fiber- or disc- shaped inclusions), the $\varepsilon_{\varphi \psi}$-VS.-sin ${ }^{2} \psi$ diagram often significantly deviates from the one obtained for a texture-free material consisting of spherical grains. 


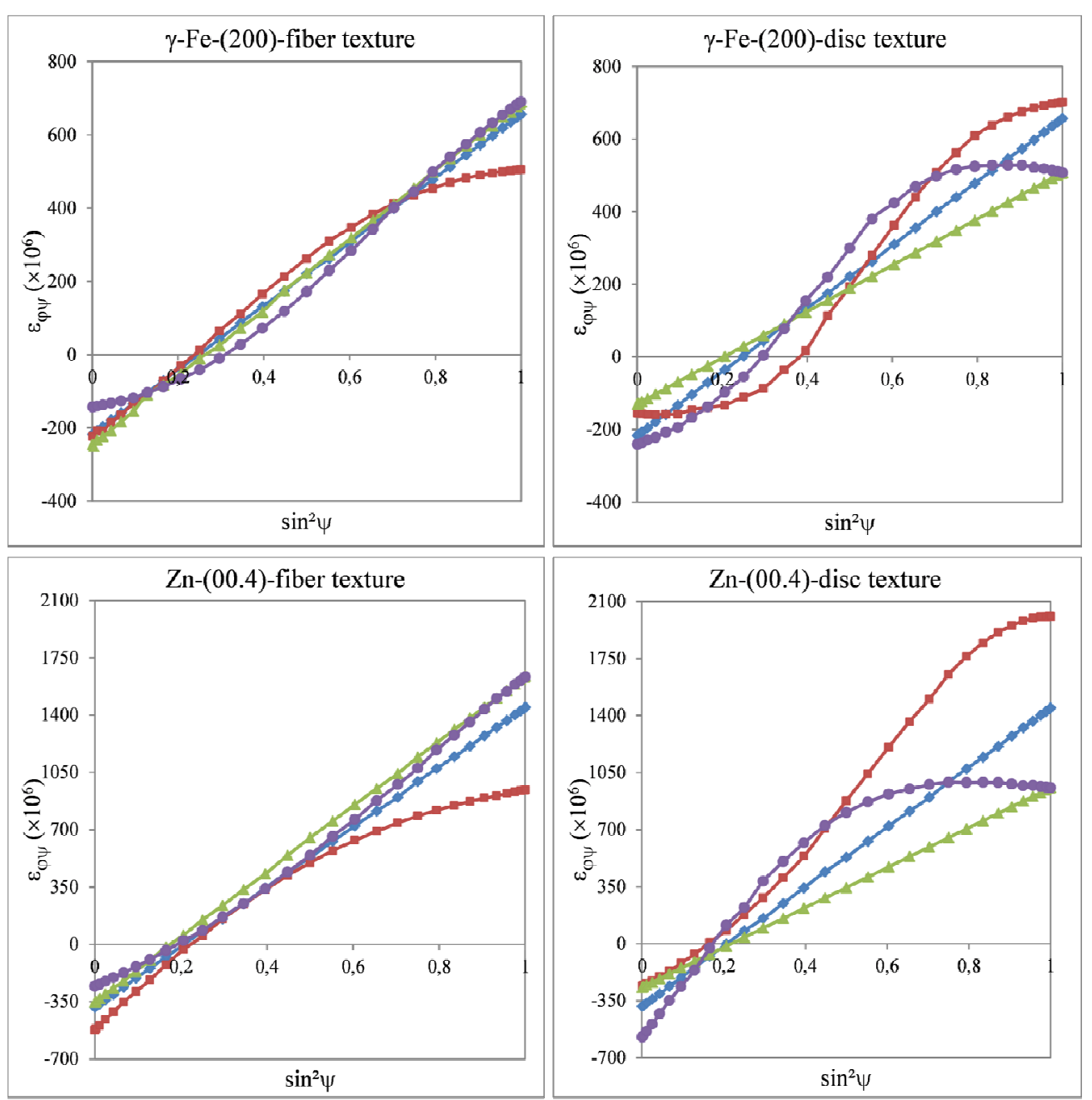

Figure $3: \varepsilon_{\varphi \psi}$-vs.-sin ${ }^{2} \psi$ diagrams for morphologically textured materials: $(--) \mathrm{C} 1 ;(\rightarrow) \mathrm{C} 2$; $(\rightarrow) \mathrm{C} 3 ;(\rightarrow)$ non-textured isotropic case with spherical grains.

\section{Discussion}

Different $\varepsilon_{\varphi \psi}$-vs.- $\sin ^{2} \psi$ distributions have been observed according to the grains shape, their orientation in the specimen and the (hkl) lattice plane analysed. Therefore, their interpretation in terms of macroscopic stresses will lead to a state of stress more or less far from the real stress in the material. To avoid all these deviations, a proper selection of reflections, less sensitive to the morphological texture, is crucial, hence the interest to study the influence of the morphological texture on the different planes used in diffraction technics. Using a linear regression $\mathrm{Asin}^{2} \psi+C$, we calculated stresses due to the texture effects which are then compared to the real state of stress in the material in terms of relative difference. A maximal relative deviation of $27.2 \%$ and $38.1 \%$ has been observed for the gamma-iron and the zinc, respectively.

Similar study was done to investigate the influence of crystallographic texture, considering a rolled texture simulated by a Taylor viscoplastic model [6] with a final plastic strain of $80 \%$ for polycrystals with cubic structure and an experimental orientation distribution function (ODF) of zircaloy-4 plate cold-rolled with a total strain rate of $47 \%$ [7] for polycrystals with hexagonal symmetry. Only a maximal relative difference of $13.3 \%$ and $11.9 \%$ has been observed for the gamma-iron and the zinc, respectively, although these crystallographic textures are very marked. When these latters were coupled with the morphological texture, a maximal combined effect of $36.6 \%$ is observed for the gamma-iron and $44.4 \%$ for the zinc. According to these results, we propose a Table (Tab. 1) to choose the (hkl) planes for stress analysis by diffraction, to minimize the effects of morphological texture or the combined effects of crystallographic and morphological textures. Tab. 1 concerns materials which single crystals are elastically anisotropic. 
Table 1: Recommendations on the choice of the lattice-plane in stresses analysis by diffraction methods according to the morphological texture; owing to the relative difference on determined stresses, some (hkl) planes should be: $(+)$ favourable for stresses analysis by diffraction methods below $10 \%$, (-) avoided, if possible, between 10 and $20 \%$ and $(\times)$ strictly avoided, up to $20 \%$.

\begin{tabular}{|c|c|c|c|c|c|c|c|c|c|c|c|c|}
\hline \multirow{3}{*}{$\begin{array}{l}\text { Lattice } \\
\text { planes }\end{array}$} & \multicolumn{6}{|c|}{$-60^{\circ} \leq \psi \leq 60^{\circ}$} & \multicolumn{6}{|c|}{$-90^{\circ} \leq \psi \leq 90^{\circ}$} \\
\hline & \multicolumn{3}{|c|}{ fiber texture } & \multicolumn{3}{|c|}{ disc texture } & \multicolumn{3}{|c|}{ fiber texture } & \multicolumn{3}{|c|}{ disc texture } \\
\hline & $\mathrm{C} 1$ & $\mathrm{C} 2$ & $\mathrm{C} 3$ & $\mathrm{C} 1$ & $\mathrm{C} 2$ & $\mathrm{C} 3$ & $\mathrm{C} 1$ & $\mathrm{C} 2$ & $\mathrm{C} 3$ & $\mathrm{C} 1$ & $\mathrm{C} 2$ & $\mathrm{C} 3$ \\
\hline \multicolumn{13}{|c|}{ polycrystal with cubic symmetry } \\
\hline$(200)$ & + & + & - & + & $\times$ & + & $\times$ & + & + & + & $x$ & - \\
\hline$(220)$ & $x$ & - & + & + & + & $x$ & - & - & + & - & + & - \\
\hline$(222)$ & $x$ & + & + & + & - & $x$ & + & + & + & - & - & + \\
\hline (311) & - & + & + & + & - & - & - & + & + & + & - & - \\
\hline$(420)$ & - & + & + & + & - & $x$ & - & + & + & + & - & - \\
\hline (331) & - & + & + & + & + & - & + & + & + & + & + & - \\
\hline (511) & - & + & - & + & $x$ & + & $x$ & + & + & + & $x$ & - \\
\hline (844) & + & + & + & + & + & + & + & + & + & + & + & - \\
\hline \multicolumn{13}{|c|}{ polycrystal with hexagonal symmetry } \\
\hline$(10.0)$ & - & + & $x$ & - & $x$ & - & + & + & $x$ & - & $x$ & + \\
\hline$(00.4)$ & + & - & + & $x$ & $x$ & - & $x$ & - & - & $x$ & $x$ & - \\
\hline$(11.0)$ & - & + & - & - & - & $x$ & + & + & - & - & - & + \\
\hline$(20.1)$ & - & + & - & - & $x$ & - & + & + & - & - & $x$ & + \\
\hline$(20.3)$ & - & - & - & + & - & $x$ & + & + & - & - & - & + \\
\hline$(11.4)$ & - & - & + & + & + & - & + & + & - & - & + & + \\
\hline$(21.3)$ & - & + & - & + & - & - & + & + & - & - & - & + \\
\hline$(30.2)$ & - & + & - & - & $x$ & - & + & + & - & - & $x$ & + \\
\hline$(10.4)$ & + & + & + & + & - & + & + & - & - & $x$ & - & + \\
\hline
\end{tabular}

\section{Conclusion}

If the single crystal of the material is almost elastically isotropic, any (hkl) plane can be used. The effects induced by the morphological texture on the stress analysis from the measured lattice strains could be neglected. In this case, the maximal relative discrepancy on the calculated stresses is small, not exceeding $10 \%$. For highly anisotropic materials which present an extreme morphological texture and possibly a crystallographic one (either weak or strong), a selection criterion was proposed to choose (hkl) planes favourable to stress analysis by diffraction, for the azimuths $\varphi=0^{\circ}$ (and $90^{\circ}$ ) commonly used in practice. Because the stress factors evaluation is far more complex than the XECs, by using appropriate (hkl) planes, XECs can be used instead of the stress factors.

\section{References}

[1] V. Hauk, Structural and Residual Stress Analysis by Nondestructive Methods, Elsevier Science edition, 1997.

[2] U. Welzel, A. Kumar and E. J. Mittemeijer, Applied Physics Letters, 95 (2009), 111907.

[3] U. F. Kocks, C. N. Tomé and H. R. Wenk, Texture and Anisotropy, Cambridge University Press edition, 1998.

[4] S. Fréour, D. Gloaguen, M. François, A. Perronnet and R. Guillén, Journal of Applied Crystallography, 38 (2005), 30-37.

[5] D. Gloaguen, G. Oum, V. Legrand, J. Fajoui and S. Branchu, Acta Materialia, 61 (2013), 57795790 .

[6] G. I. Taylor, Journal of the Institute of Metals, 62 (1938), 307-324.

[7] D. Gloaguen, T. Berchi, E. Girard and R. Guillén, Acta Materialia, 55 (2007), 4369-4379. 